\title{
TRANSICIÓN ALIMENTARIA EN POBLACIONES ORIGINARIAS DEL NORTE DE TIERRA DEL FUEGO DURANTE EL PERÍODO MISIONAL SALESIANO: UNA APROXIMACIÓN ISOTÓPICA
}

\section{DIETARY TRANSITION IN NATIVE POPULATIONS OF NORTHERN TIERRA DEL FUEGO DURING THE SALESIAN MISSIONARY PERIOD: AN ISOTOPIC APPROACH}

Luciano O. Valenzuela1,2* , Pamela García Laborde², Manuel D. D’Angelo del Campo ${ }^{2,3}$ y Ricardo A. Guichón ${ }^{1,2}$

\begin{abstract}
${ }^{1}$ Consejo Nacional de Investigaciones Científicas y Técnicas (CONICET). Argentina
${ }^{2}$ Laboratorio de Ecología Evolutiva Humana (LEEH). Núcleo de Estudios Interdisciplinarios sobre Poblaciones Humanas de Patagonia Austral (NEIPHPA). Unidad de Enseñanza Universitaria de Quequén. Facultad de Ciencias Sociales. Universidad del Centro de la Provincia de Buenos Aires. Quequén. Argentina

${ }^{3}$ Laboratorio de Poblaciones de Pasado (LAPP). Departamento de Biología. Facultad de Ciencias. Universidad Autónoma de Madrid (UAM). Madrid. España
\end{abstract}

\section{PALABRAS CLAVE contacto interétnico; isótopos estables; Selk'nam}

RESUMEN La misión salesiana La Candelaria funcionó de 1893 a 1928 en el norte de Tierra del Fuego, aunque su cementerio fue usado hasta 1948. Las fuentes históricas sugieren que los nativos que la habitaron sufrieron fuertes cambios en su dieta con respecto a los cazadoresrecolectores de la zona. El cambio estaría caracterizado por un gran incremento en el consumo de harina, arroz, papas y azúcar, que remplazan al guanaco como fuente principal de alimento. Nuestro objetivo es evaluar la transición alimentaria de los habitantes de la misión a través del análisis de isótopos estables de carbono y nitrógeno sobre huesos (colágeno, apatita) y dientes (dentina, esmalte) de 23 individuos exhumados del cementerio. Los valores de $\delta^{15} \mathrm{~N}$ en colágeno $\left(\delta^{15} \mathrm{Nc}\right)$ y de $\delta^{13} \mathrm{C}$ tanto en colágeno $\left(\delta^{13} \mathrm{C}_{\mathrm{c}}\right)$ como en apatita $\left(\delta^{13} \mathrm{C}_{\mathrm{a}}\right)$ de individuos del cementerio resultaron más bajos que los valores publicados para la región para períodos pre-contacto. Dentro del cementerio, los valores de $\delta^{15} \mathrm{~N}_{\mathrm{c}}$ y de $\delta^{13} \mathrm{C}_{\mathrm{a}}$ resultaron más bajos que los de $\delta^{15} \mathrm{~N}_{\mathrm{d}}$ (dentina) y de $\delta^{13} \mathrm{C}_{\mathrm{e}}$ (esmalte), pero no hubo diferencias entre valores de $\delta^{13} \mathrm{C}_{\mathrm{c}}$ y $\delta^{13} \mathrm{C}_{\mathrm{d}}$. Los valores de $\delta^{15} \mathrm{~N}_{\mathrm{d}}$ y $\delta^{13} \mathrm{C}_{\mathrm{d}}$ resultaron significativamente más bajos que los valores de colágeno para períodos pre-contacto. Estos resultados son consistentes con la hipótesis planteada por las fuentes históricas y sugieren un desequilibrio en la dieta, con una mayor proporción de carbohidratos y una menor proporción de proteínas animales en los habitantes de la misión con relación a los cazadores-recolectores de Tierra del Fuego de períodos pre-contacto. Rev Arg Antrop Biol 21(1), 2019. doi:10.17139/raab.2019.0021.01.02

\section{KEY WORDS inter-ethnic contact; stable isotopes; Selk'nam}

ABSTRACT The Salesian mission La Candelaria was run from 1893 to 1928 in the north of Tierra del Fuego, but its cemetery was in use until 1948. Historical sources suggest that the natives living there underwent marked dietary changes with respect to the hunter-gatherers of the area. The change can be characterized by a large increase in the consumption of flour, rice, potatoes, and sugar, in replacement of guanaco, as their main food source. Our objective is to evaluate the dietary transition experienced by the inhabitants of the mission through the analysis of stable isotopes of carbon and nitrogen on bones (collagen, apatite) and teeth (dentin, enamel) of 23 individuals exhumed from the cemetery. The $\delta^{15} \mathrm{~N}$ values in collagen $\left(\delta^{15} \mathrm{~N}_{c}\right)$, and $\delta^{13} \mathrm{C}$ values both in collagen $\left(\delta^{13} \mathrm{C}_{\mathrm{c}}\right)$ and in apatite $\left(\delta^{13} \mathrm{C}_{\mathrm{a}}\right)$ of samples from the cemetery were lower than the values published for the region in pre-contact periods. Within the cemetery, the $\delta^{15} \mathrm{~N}_{\mathrm{c}}, \delta^{13} \mathrm{C}_{\mathrm{c}}$ and $\delta^{13} \mathrm{C}_{\mathrm{a}}$ values were lower than those of $\delta^{15} \mathrm{~N}_{\mathrm{d}}$ (dentin) and $\delta^{13} \mathrm{C}_{\mathrm{e}}$ (enamel), but there were no differences between $\delta^{13} \mathrm{C}_{\mathrm{c}}$ and $\delta^{13} \mathrm{C}_{\mathrm{d}}$ values. The $\delta^{15} \mathrm{~N}_{\mathrm{d}}$ and $\delta^{13} \mathrm{C}_{\mathrm{d}}$ values were significantly lower than the isotope values of collagen for pre-contact periods. The results are consistent with the hypothesis raised by histori- cal sources and suggest an imbalance in the diet, with a higher proportion of carbohydrates and a lower proportion of animal proteins in the inhabitants of the mission in comparison with the hunter-gatherers of Tierra del Fuego of pre-contact periods. Rev Arg Antrop Biol 21(1), 2019. doi:10.17139/raab.2019.0021.01.02

Financiamiento: Agencia Nacional de Promoción Científica y Tecnológica: PICT 01520 (2007-2010) y PICT 0575 (2011-2013).

*Correspondencia: Luciano O. Valenzuela. Laboratorio de Ecología Evolutiva Humana. Unidad de Enseñanza Universitaria de Quequén. Facultad de Ciencias Sociales. Universidad del Centro de la Provincia de Buenos Aires. Calle 508 NN$^{\circ} 881$. 7631 Quequén. Argentina.

E-mail: lucianoovalenzuela@gmail.com

Recibido 06 Diciembre 2017; aceptado 19 Marzo 2018

doi:10.17139/raab.2019.0021.01.02 
El proceso de contacto entre europeos e indígenas en Patagonia Austral (PA, la región de Argentina y Chile ubicada al sur del río Santa Cruz, aproximadamente $50^{\circ} \mathrm{S}$ ) debe ser reconocido como un proceso complejo prolongado en el tiempo (Borrero, 1992, 2001a; Guichón, Suby, Casali, y Fugassa, 2006; Ramenofsky, 1988). El contacto directo, aquel continúo y permanente, principalmente desde mediados del siglo XIX es sin lugar a dudas el proceso que introdujo los mayores cambios en la vida y la salud de los nativos de la PA (Borrero, 2001b; Casali, 2011; Casali, Fugassa, y Guichón, 2006; Guichón et al., 2006). En Tierra del Fuego el gran desarrollo de la ganadería ovina a partir de 1885 y el cercado de territorios acarrearon la escasez del guanaco (Lama guanicoe), recurso básico de los nativos Selk'nam, en la región (Borrero, 2001b; Casali, 2011). Conjuntamente, la actividad ganadera alteró la territorialidad y los movimientos de los grupos de indígenas, produciendo un incremento en los conflictos inter e intraétnicos y también generó escasez de alimento y materias primas (Casali, 2011; Casali et al., 2006; Guichón et al., 2006). Por tanto, los grupos de nativos se vieron forzados a cambiar sus hábitos alimenticios, de apropiación de recursos y de movilidad (Borrero, 2001b; Casali, 2011; Casali et al., 2006). Paralelamente, a partir de 1880 se produjo una concentración de grupos de nativos en estancias y misiones que implicaron modificaciones drásticas en los modos de vida (Borrero, 2001b; Casali, 2011).

Como parte de la estrategia de colonización, la Misión Salesiana "Nuestra Señora de La Candelaria" fue establecida en 1893 cerca de la actual ciudad de Río Grande en el norte de Tierra del Fuego (Casali, 2011; García Laborde et al., 2010). La Misión funcionó entre 1893 y 1928 , aunque su cementerio fue usado por habitantes de la región hasta 1948 (Casali, 2011; García Laborde, 2017; García Laborde et al., 2010). La vida en las misiones representó para los nativos una situación contrastante con el modo de vida pre-contacto.

Los trabajos en la Misión Salesiana, tanto sobre fuentes escritas como sobre el registro bioarqueológico del cementerio, han generado importante información acerca de los cambios en las condiciones de vida producto del contacto inter-étnico en la región. Casali (2011) presenta un detallado análisis de los registros históricos y reconstruye los diferentes períodos de la misión integrando variables tales como: número de habitantes (y densidad), número de defunciones (y sus causas), movilidad de sus habitantes, dieta y actividades. De acuerdo a este análisis, y en cuanto a la dieta, se postula un gran incremento en el consumo de hidratos de carbono (particularmente harina, arroz, papas y azúcar) hasta el año 1903, a partir del cual existe un aumento en el consumo de ovejas, aunque el consumo de harina se mantiene elevado (Casali, 2011). Además, el período 1900-1903 estuvo caracterizado por una mayor densidad poblacional y un aumento en el número de defunciones (Casali, 2011). Estos cambios representan alteraciones en el balance de proteínas e hidratos de carbono para los individuos que habitaron la misión que podrían ser corroborados con indicadores de dieta como los isótopos estables. El estudio por isótopos estables posibilita obtener información de la dieta y ambiental, ya que en la composición isotópica del tejido del consumidor (hueso, dientes, pelo, uñas) se refleja la señal isotópica de sus alimentos.

\section{Uso de isótopos estables para el estudio de la dieta y uso de recursos}

Las moléculas de carbono y nitrógeno presentes en los tejidos humanos son derivadas exclusivamente de la dieta, y las proporciones de sus isótopos estables reflejan aquellas de los productos consumidos (Boutton, Lynott, y Bumsted, 1991; DeNiro y Epstein, 1978, 1981; DeNiro y Schoeninger, 1983; Fry, 2006; Peterson y Fry, 1987; Petzke, Boeing, Klaus, y Metges, 2005; Petzke, Fuller, y Metges, 2010; Schwarcz y Schoeninger, 1991). Las proporciones de los isótopos estables del carbono $\left(\delta^{13} \mathrm{C}\right)$ reflejan fielmente las proporciones de los isótopos de carbono de la fuente de alimentación original y han sido utilizadas extensivamente como indicadoras de la proporción de plantas $\mathrm{C}_{3}$ (por ejemplo, gramíneas de zonas templadas, frutas, verduras) o plantas $\mathrm{C}_{4}$ (por ejemplo, gramíneas tropicales, maíz, caña de azúcar) en la dieta humana, ya sea consumidas directamente como alimento básico o indirectamente a través de la alimentación animal (Fry, 2006; Kellner y Schoeninger, 2007; Kelly, 2000; Schwarcz y 
Schoeninger, 1991). Además, en ciertas regiones donde existe un gran contraste entre plantas $\mathrm{C}_{3} \mathrm{y}$ alimento de origen marino, los valores de $\delta^{13} \mathrm{C}$ en tejidos óseos se han usado para diferenciar el consumo de recursos de origen terrestre y costeros (Fry, 2006; Kellner y Schoeninger, 2007; Peterson y Fry, 1987; Schwarcz y Schoeninger, 1991). A su vez, la medición de los isótopos estables del carbono en la fracción inorgánica de huesos (apatita) y dientes (esmalte) reflejan un promedio de la dieta total metabolizada para energía (hidratos de carbono, grasas y proteínas), mientras que los valores de $\delta^{13} \mathrm{C}$ de la fracción orgánica de huesos (colágeno) y dientes (dentina) reflejan principalmente el consumo de proteínas (Clementz, Fox-Dobbs, Wheatley, Koch, y Doak, 2009; Fry, 2006; Kellner y Schoeninger, 2007). Contrario al carbono, las moléculas de nitrógeno solo son obtenidas al consumir proteínas. Las proporciones de isótopos estables del nitrógeno $\left(\delta^{15} \mathrm{~N}\right)$ en los tejidos de un consumidor son generalmente más elevadas en relación a su dieta (DeNiro y Epstein, 1981; DeNiro y Schoeninger, 1983; Kelly, 2000). En mamíferos esto se debe a la retención preferencial del isótopo más pesado $\left({ }^{15} \mathrm{~N}\right)$ durante la excreción (DeNiro y Epstein, 1981; Fry, 2006; Sponheimer et al., 2003). Debido a esta retención o enriquecimiento, los valores de $\delta^{15} \mathrm{~N}$ de los consumidores aumentan con cada nivel trófico en forma predecible. Este permite utilizar a los valores de $\delta^{15} \mathrm{~N}$ de los tejidos analizados como indicadores de la proporción de proteínas animales y vegetales en la dieta de omnívoros (DeNiro y Schoeninger, 1983; Fry, 2006; Hedges y Reynard, 2007; Petzke et al., 2005)

\section{Estudios previos con isótopos estables en Patagonia Austral}

Los estudios en PA han utilizado isótopos estables del carbono y nitrógeno para evaluar distintos aspectos de la dieta y la vida de las poblaciones pre-contacto. Entre las investigaciones más recientes para la región austral de la Patagonia, es posible mencionar los trabajos de Borrero, Barberena y sus coautores que describen muy bien el uso de los isótopos estables, junto a otras líneas de evidencia (por ejemplo: restos líticos), para estimar dietas, uso de hábitats y rango de acción (home range) en pobladores del sur de la Provincia de Santa Cruz (por ejemplo: Barberena, 2004; Borrero et al (2001); Borrero y Barberena, 2006; Borrero, Barberena, Franco, Charlin, y Tykot, 2009). Estos trabajos se centran en distinguir el uso y consumo de recursos terrestres (principalmente guanaco, Lama guanicoe) de recursos marinos (principalmente lobo marino, Otaria flavescens y Artocephalus australis). Además, demuestran home ranges relativamente acotados, pero también diferencias en las estrategias del uso de hábitat entre asentamientos humanos no muy distantes.

En Tierra del Fuego, la variabilidad de los patrones dietarios y el uso de recursos han sido estudiados por varios autores utilizando isótopos estables como marcadores dietarios desde hace casi 30 años (Borrero y Barberena, 2006; Borrero et al., 2001; 2009; Guichón, Borrero, Prieto, Cárdenas, y Tykot, 2001; Guichón et al., 2006; Panarello, Zangrando, Tessone, Kozameh, y Testa, 2006; Santiago, Salemme, Suby, y Guichón, 2011; Schinder y Guichón, 2003; Schinder, Guichón, Comparatore, y Burry, 2003; Suby y Guichón, 2009; Tafuri et al., 2017; Tessone, Zangrando, Valencio, y Panarello, 2003; Yesner, Figuerero Torres, Guichón, y Borrero, 1991; Yesner, Torres, Guichon, y Borrero, 2003; Zangrando, Panarello, y Piana, 2014; Zangrando et al., 2004). Estos análisis evidencian una variación geográfica expresada en dietas fundamentalmente terrestres en el norte de la Isla Grande, con uso del guanaco como fuente primaria, y dietas principalmente marinas para los habitantes del Canal de Beagle y Península Mitre. Sin embargo, estos marcadores también dan cuenta de estrategias más flexibles a la hora del uso de los recursos que lo postulado por los recuentos etnográficos (Gusinde, 1990). Por ejemplo, en un meta-análisis Schinder et al. (2003) establecen que sobre un total de 36 individuos de tres regiones (Norte de la Isla, Sudeste de la Isla y la costa del Canal de Beagle) las dietas mixtas representan el 44\%, mientras que el resto pueden clasificarse como dietas puramente terrestres $(32 \%)$ o marinas $(23 \%)$.

Planteamos entonces, analizar cambios en la dieta vinculados al proceso de contacto interétnico en el norte de Tierra del Fuego a través del uso de isótopos estables (carbono y nitrógeno) en restos humanos. Específicamente, evaluaremos las diferencias en los valores isotópicos, 
estimadores de dieta, entre individuos recuperados en el cementerio de la Misión Salesiana "Nuestra Señora de La Candelaria", asumidos como pertenecientes al periodo temporal misional, e individuos de períodos pre-contacto recuperados en PA, particularmente en el territorio norte de Tierra del Fuego. Las investigaciones a partir de fuentes históricas (Casali, 2011; Martucci, 2016) nos permiten plantear como hipótesis un descenso del consumo proporcional de proteína animal (p.e. guanaco) como respuesta al gran incremento de los hidratos de carbono (p.e. plantas $\mathrm{C}_{3}$ ). En este sentido, se espera obtener valores de $\delta^{15} \mathrm{~N}$ más bajos para individuos de la misión, así como también valores de $\delta^{13} \mathrm{C}$ más bajos como respuesta a esta modificación de la dieta.

\section{MATERIAL Y MÉTODOS}

\section{Restos humanos}

Los restos humanos recuperados en el cementerio de La Misión Salesiana y las excavaciones correspondientes han sido descriptos en publicaciones previas (García Laborde, 2011, 2017; García Laborde et al., 2010). Los detalles de esta muestra (perfiles biológicos, estudios palepatológicos y tafonómicos) se pueden ver en esas publicaciones; sin embargo, para este trabajo podemos decir que la determinación de sexo y estimación de edad en adultos se llevaron a cabo siguiendo los métodos propuestos por (Buikstra y Ubelaker, 1994). En la determinación del sexo se consideró, en los casos posibles, la morfología del esqueleto completo pero prestando mayor atención a la morfología de la pelvis (arco ventral, la concavidad subpúbica, la superficie de la rama isquiopúbica, la escotadura ciática y el surco preauricular) y el cráneo (cresta nucal, proceso mastoide, margen supraorbital, la glabela y la eminencia mentoniana) (Bass, 1995; Buikstra y Mielke, 1985; Buikstra y Ubelaker, 1994; Ubelaker, 1999). La edad se estimó teniendo en cuenta la metamorfosis de la sínfisis púbica, el extremo esternal de la cuarta costilla, la superficie auricular de la pelvis, la clavícula y suturas maxilares (Brooks y Suchey, 1990; Mann, Jantz, Bass, y Willey, 1991; Meindl y Loveyoy, 1989; Szilvassy, 1988). En cuanto a la edad de los subadultos, la determinación se basó en el desarrollo y erupción dental, la lon- gitud máxima de los huesos largos, el largo y el ancho máximo de los huesos craneales, así como también la fusión ósea (Cunningham, Scheuer, y Black, 2000; Fazekas y Kosa, 1978; Krogman y İşcan, 1986; Scheuer, Black, y Schaefer, 2008; Ubelaker, 1999). Para la determinación de sexo en subadultos se aplicó el método propuesto por Schutkowski (1993) que enfatiza la observación del ilion y la mandíbula. En los casos en que la preservación de los elementos lo permitió, se utilizó también la osteometría de los huesos largos, concretamente la longitud de huesos como fémur, tibia, peroné, húmero, cúbito y radio, que luego se compararon con estándares presentados por Cunningham et al. (2000). El estado de preservación se estableció relacionando la integridad ósea y pérdida ósea siguiendo criterios establecidos por otros autores (Bello, Thomann, Signoli, Dutour, y Andrews, 2006; González, 2007). Para más detalles tafonómicos sobre los restos humanos del cementerio ver García Laborde, Guichón, y González, (2015). Concretamente en este trabajo se presentan análisis de 23 individuos, de los cuales 16 son adultos (6 femeninos y 10 masculinos) y 7 corresponden a la categoría de subadultos, es decir menores de 18 años (3 femeninos, 3 masculinos y 1 individuo que no pudo determinarse el sexo)

\section{Preparación de muestras}

La preparación de huesos y dientes para su análisis isotópico siguió protocolos y procedimientos estándares (Ambrose, 1990; Ambrose y DeNiro, 1986; DeNiro, 1985; Luz y Kolodny, 1985; Yoder y Bartelink, 2010). Huesos: diez muestras de costillas fueron enviadas directamente para ser procesadas y analizadas en el Center for Applied Isotope Studies (CAIS), University of Georgia. De acuerdo con los reportes del CAIS, el hueso se limpió con un cepillo de alambre y se lavó usando un baño ultrasónico. Después de la limpieza, el hueso seco se trituró suavemente en pequeños fragmentos. Para el análisis de isótopos de colágeno, el hueso triturado se trató con ácido clorhídrico $(\mathrm{HCl}) 1 \mathrm{~N}$ a $4^{\circ} \mathrm{C}$ durante 24 horas. El residuo se filtró, se enjuagó con agua desionizada bajo condiciones ligeramente ácidas $(\mathrm{pH}=3)$, se calentó a $80^{\circ} \mathrm{C}$ durante 6 horas para disolver colágeno y dejar sustancias húmicas en el precipitado. La solución de colágeno luego fue filtrada y liofilizada. 
Para el análisis de isótopos de bioapatita, el hueso triturado se trató con ácido acético $1 \mathrm{~N}$ diluido para eliminar los carbonatos secundarios. La muestra químicamente limpia se hizo reaccionar entonces bajo vacío con $\mathrm{HCl} 1 \mathrm{~N}$ para disolver el mineral óseo y liberar dióxido de carbono de la bioapatita. Dientes: se tomaron muestras de dientes correspondientes a 23 individuos utilizando un taladro de alta velocidad $\left(\right.$ Dremel $\left.^{\circledR}\right)$ que permitió realizar pequeñas perforaciones, obtener polvo y disminuir el daño del diente. Todos los dientes fueron muestreados en los laterales de la corona. El procesamiento inicial de las muestras para su análisis isotópico se realizó en el Laboratorio de Ecología Evolutiva Humana ubicado en la Subsede Quequén de la UNCPBA, y los análisis isotópicos se realizaron en el Stable Isotope Ratio Facility for Environmental Research (SIRFER.), University of Utah. El polvo de dentina fue sometido a una desmineralización liviana usando $\mathrm{HCl} 0.1 \mathrm{~N}$ por 24 horas a $4^{\circ} \mathrm{C}$. El polvo de esmalte fue sujeto a una reacción de oxidación con $30 \%$ peróxido de hidrógeno durante 30 minutos, seguido por tres lavados con agua doble destilada (ADD), luego un lavado de 30 minutos con $0.1 \mathrm{M}$ acetato de sodio para eliminar carbonatos secundarios. Por último, se lavó tres veces con ADD y se dejó secar a temperatura ambiente.

Todos los análisis se realizaron en un Isotope Ratio Mass Spectrometer en modo de flujo continuo. Los datos son reportados en la notación estándar $\delta$ (delta) en unidades de partes por mil (\%o). Esta notación representa diferencias relativas en la tasa de isótopos estables de una muestra con respecto a un material estándar (por ejemplo: para el carbono): $\delta^{13} \mathrm{C}(\%)=\left[\left(\mathrm{R}_{\text {muestra }} / \mathrm{R}_{\text {estándar }}-1\right) \times 1000\right]$, donde $\mathrm{R}$ representa la tasa del isótopo pesado con respecto al liviano $\left({ }^{13} \mathrm{C} /{ }^{12} \mathrm{C}\right)$. Los valores de $\delta^{13} \mathrm{C}$ se expresan con respecto a la escala $\mathrm{V}-\mathrm{PDB}$ con error menor a $0.1 \%$, mientras que los valores de $\delta^{15} \mathrm{~N}$ se expresan con respecto a la escala AIR con error menor a $0.2 \%$. Los errores fueron estimados luego de mediciones repetidas de estándares internos de los laboratorios correspondiente.

Los restos recuperados en la misión corresponden a individuos que murieron después de 1897 (año de apertura del cementerio), por lo tanto debería aplicarse una corrección por el "efecto Suess" a los valores de $\delta^{13} \mathrm{C}$ para dar cuenta de la disminución global en la razón ${ }^{13} \mathrm{C} /{ }^{12} \mathrm{C}$ del $\mathrm{CO}_{2}$ atmosférico durante el último siglo y medio (Francey et al., 1999). Sin embargo, no podemos corregirlos directamente ya que el año de muerte para cada individuo es desconocido. Por lo tanto, presentamos los datos "sin corregir" y discutimos esto cuando es apropiado.

\section{Valores isotópicos de la bibliografía}

Para poner en contexto los datos generados en esta investigación se comparan los mismos con datos isotópicos publicados para la región de PA. Para incluir o excluir valores isotópicos se siguió el siguiente criterio:

1- Individuos recuperados de sitios localizadas al sur del paralelo $50^{\circ} \mathrm{S}$.

2- En los casos que existen diferentes valores para una misma muestra (mismo individuo y mismo tejido) se dio prioridad a los valores reportados en estudios realizados directamente para isótopos estables y no aquellos medidos como parte del proceso de datación por ${ }^{14} \mathrm{C}$.

3- Los individuos se asignaron a seis regiones de PA siguiendo la división usada por Borrero et al. (2001); Cordillera Última Esperanza (CUE), Interior del Continente (IC), San Gregorio Brunswick (SGB), Norte de la Isla Grande (NIG), costa Canal de Beagle (CB) y Península Mitre o Sudeste de Isla Grande (PM). En la Figura 1 se representan estas seis regiones.

\section{Estadística}

Debido a los pequeños tamaños muestrales se trabajó con análisis no paramétricos para comparar grupos; Kruskal-Wallis y test de suma de rangos Wilcoxon (también llamado Wilcoxon-Mann-Whitney). Las correlaciones del total de los datos se realizaron usando métodos paramétricos (Coeficiente de correlación Pearson). Todos los análisis estadísticos y representaciones gráficas fueron realizadas usando $\mathrm{R}$ (R Development Core Team, 2008).

\section{RESULTADOS}

Trabajamos sobre 23 individuos exhumados del cementerio de la misión. En la tabla 1 se presentan los valores isotópicos para estos individuos, conjuntamente con información de sus perfiles biológicos. En cuanto a los análisis 


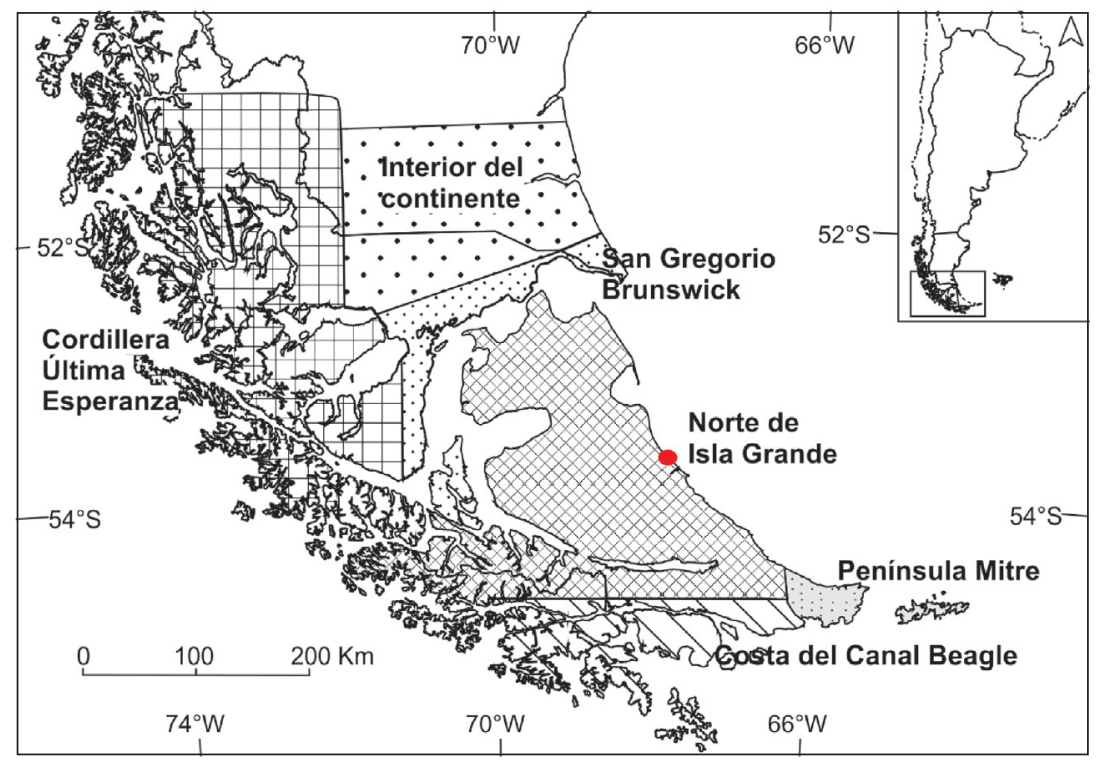

Fig. 1. Mapa resaltando las seis regiones de Patagonia Austral discutidas en este trabajo. Las regiones son Cordillera Última Esperanza (CUE), Interior del Continente (IC), San Gregorio Brunswick (SGB), Norte de Isla Grande (NIG), costa Canal de Beagle (CB) y Península Mitre o Sudeste de Isla Grande (PM). Modificado de Borrero et al. (2001). La ubicación la Misión Salesiana se encuentra marcada con un círculo rojo.

de isótopos estables en huesos, el laboratorio CAIS reportó solo las razones $\mathrm{C} / \mathrm{N}$ por lo que la evaluación de la preservación del colágeno es limitada. Sin embargo, estas razones se encuentran cercanas al rango esperado para muestras prehistóricas bien preservadas (2.9 - 3.6; según Ambrose, 1990) o dentro del mismo (2.7 - 3.6; según Schwarcz y Schoeninger, 1991). En cuanto al análisis de dientes, realizado en SIRFER, vemos que uno de los individuos [E15-16 (1)] presenta un porcentaje de nitrógeno en dentina muy bajo (6.8\%), aunque el porcentaje de carbono se encuentra dentro del rango aceptado; generando una alta razón C/N (4.9). Por lo tanto, los valores de isótopos estables medidos sobre dentina para esta muestra no son usados en los análisis. El resto de los valores para dentina se encuentran dentro de los rangos esperados.

De los 23 individuos, dos [E 15-16 (3) y E-D 10] poseen haplogrupo mitocondrial perteneciente a un linaje europeo, el resto a linajes nativos americanos [Tabla 1; (Motti et al., 2015)]. $\mathrm{Al}$ tener linajes maternos europeos, no se los incluye en los análisis y comparaciones estadísticas. Además, en las comparaciones estadísticas no se incluye el individuo E 14-15 (1) ya que la muestra proviene de un diente deciduo (segundo molar) y por tanto presentaría el efecto de la dieta materna y la lactancia, lo cual es evidente en su alto valor de $\delta^{15} \mathrm{~N}_{\mathrm{d}}(16.2 \%)$ en comparación con el resto de la muestra.

Tanto hombres como mujeres del cementerio presentan valores isotópicos similares. No se detectó ninguna diferencia significativa en las comparaciones de los valores isotópicos en hueso o diente entre sexos (test Wilcoxon-MannWhitney; $\mathrm{p}>0.05$ ). Todas las comparaciones isotópicas entre grupos de edad fueron no significativas (análisis Kruskal-Wallis; $p>0.05$ ), aunque esta última comparación se ve muy afectada por el bajo número de muestras en cada categoría etaria. Por tanto, en el resto de las comparaciones se trabajó con la muestra del cementerio como un todo.

El análisis bibliográfico para PA dio cuenta de valores isotópicos para 140 individuos. En el Material suplementario 1 se presentan tabulados los valores isotópicos correspondientes y en el Material suplementario 2 las referencias bibliográficas consultadas. Tomando esta base de datos, se observan correlaciones altas y significativas tanto entre los valores de nitrógeno $\left(\delta^{15} \mathrm{~N}_{\mathrm{c}}\right)$ y carbono $\left(\delta^{13} \mathrm{C}_{\mathrm{c}}\right)$ para colágeno $(\mathrm{r}=0.86$, $\mathrm{p}<0.01$, g.l. $=115$; Fig. 2) como entre $\delta^{13} \mathrm{C}_{\mathrm{c}} \mathrm{y}$ 
carbono para apatita $\left(\delta^{13} \mathrm{C}_{\mathrm{a}} ; \mathrm{r}=0.66, \mathrm{p}<0.01\right.$, g.1.=45; Fig. 2). Los diferentes grupos analizados (determinados por región y época) ocupan distintas regiones de estas distribuciones (Fig. 2). Los valores de isótopos estables de colágeno $\left(\delta^{13} \mathrm{C}_{\mathrm{c}}\right.$ y $\left.\delta^{15} \mathrm{~N}_{\mathrm{c}}\right)$ para muestras pre-contacto resultaron significativamente diferentes entre las seis regiones planteadas; para $\delta^{13} \mathrm{C}_{\mathrm{c}}$ : Kruskal-Wallis $X^{2}=39.5$, g.1. $=4, \mathrm{p}<0.001$; para $\delta^{15} \mathrm{~N}_{\mathrm{c}}$ : KruskalWallis $X^{2}=24.6$, g.l. $=4, \mathrm{p}<0.001$ (Fig. 2). Sin embargo, los valores de isótopos de carbono en apatita $\left(\delta^{13} \mathrm{C}_{\mathrm{a}}\right)$ no resultaron diferentes entre regiones (Kruskal-Wallis $X^{2}=6.9$, g.l. $=4, \mathrm{p}=0.14$ ). Este último resultado se debe quizás al reducido número de muestras representadas en cada región (Material suplementario 1). El número de muestras adjudicables ciertamente a lo que se puede considerar momentos de contacto o postcontacto para el Norte de la Isla Grande (NIG) de Tierra del Fuego (y que no sean de la Misión Salesiana) es muy bajo (Fig. 2; Material suplementario 1) como para realizar comparaciones estadísticas; sin embargo, sus valores isotópicos no parecen desviarse de las distribuciones isotópicas de NIG pre-contacto (Material suplementario 1).

Se realizó una comparación a través del test de suma de rangos Wilcoxon para muestras no pareadas entre las muestras pre-contacto del NIG y las muestras del cementerio (Fig. 2). Los valores de $\delta^{15} \mathrm{~N}_{\mathrm{c}}$ resultaron significativamente más bajos ( $\mathrm{W}=147.5, \mathrm{p}<0.001)$ para las muestras del cementerio $(9.7 \pm 2.4 \%, \mathrm{n}=9)$ que para las muestras NIG pre-contacto (13.6 $\pm 1.7 \%$, $\mathrm{n}=18$ ). De la misma manera, los valores de $\delta^{13} \mathrm{C}_{\mathrm{c}}$ entre los dos grupos resultaron significativamente diferentes $(\mathrm{W}=122, \mathrm{p}=0.04)$, con valores más bajos para el cementerio $(-18.9 \pm 1.0 \%, \mathrm{n}=9)$ que para NIG $(-17.4 \pm 2.1 \%$ o, $n=18)$. En cuanto a los valores $\delta^{13} \mathrm{C}_{\mathrm{a}}$, los mismos resultaron más bajos para el cementerio $(-15.6 \pm 1.1 \%, \mathrm{n}=9)$ que para NIG $(-14.0 \pm 1.6 \%$, $\mathrm{n}=6)$ aunque el resultado del test fue marginal $(\mathrm{W}=44, \mathrm{p}=0.048)$.

La comparación entre los valores isotópicos de dientes y huesos de individuos de la misión resultó en ciertas diferencias significativas (Fig. 3). Al comparar la fracción orgánica (colágeno y dentina) entre ambos tipos de muestras encontramos que los valores de $\delta^{15} \mathrm{~N}_{\mathrm{c}}$ resultaron más bajos $(\mathrm{W}=26.5, \mathrm{p}=0.004)$ que los valores de isótopos de nitrógeno en dentina 


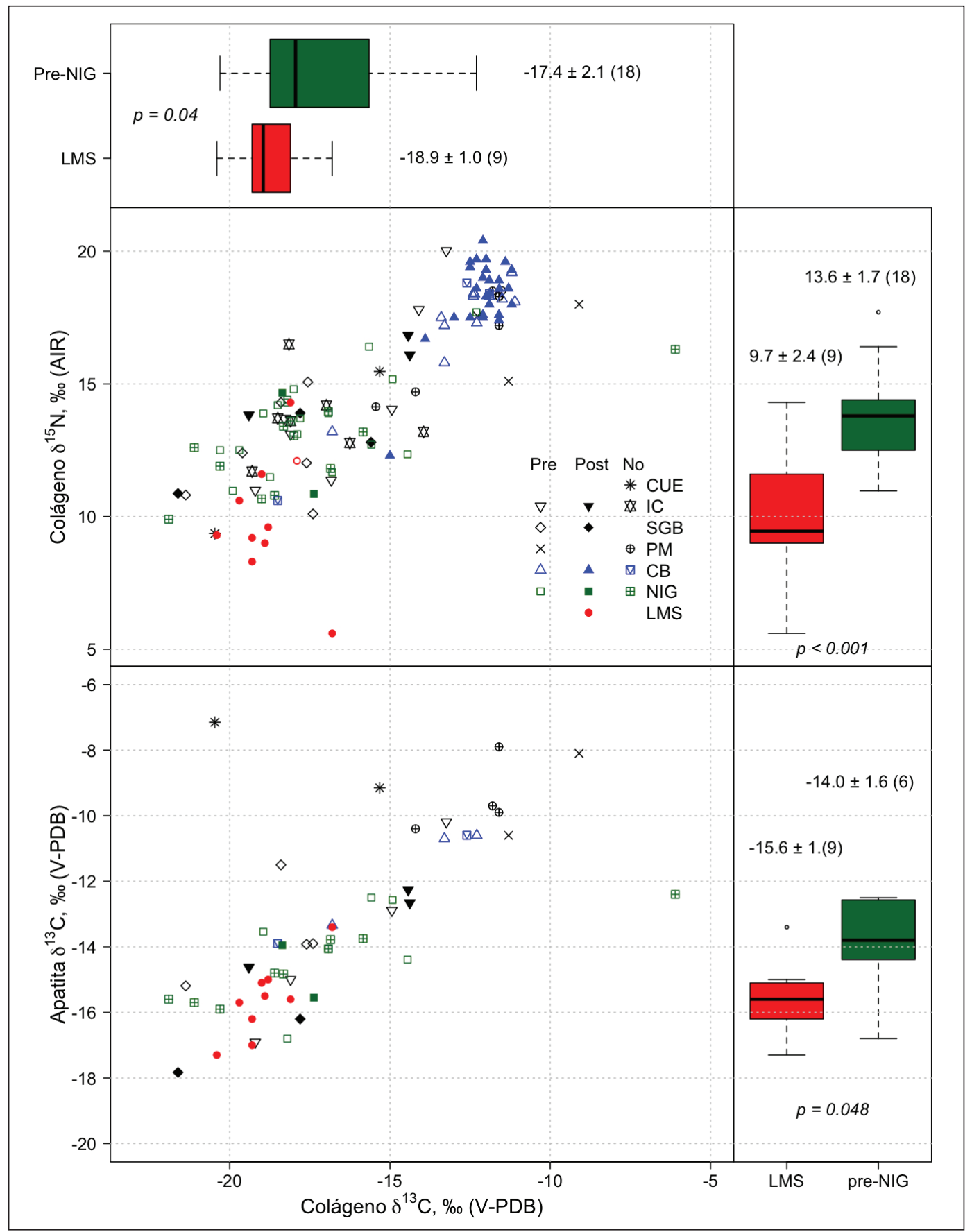

Fig. 2. Valores de $\delta^{15} \mathrm{~N}$ y $\delta^{13} \mathrm{C}$ de colágeno, y $\delta^{13} \mathrm{C}$ de apatita para individuos de PA divididos por regiones y por momentos Pre-contacto (Pre), Post-Contacto (Post) y aquellos que aún no han sido asignados a ninguno de estos momentos (No). Las regiones son Cordillera Última Esperanza (CUE), Interior del Continente (IC), San Gregorio Brunswick (SGB), Norte de Isla Grande (NIG), costa Canal de Beagle (CB) y Península Mitre o sudeste de Isla Grande (PM). Además, también se presentan los valores de isótopos de las muestras del cementerio de la Misión Salesiana (LMS). Los boxplots marginales comparan los valores de isótopos estables de la misión con los valores de muestras pre-contacto para NIG (Pre-NIG). Además, se prestan las medias $\pm 1 \sigma$ (tamaño muestral), y el valor de $\mathrm{p}$ luego del test de suma de rangos Wilcoxon para muestras no pareadas. 


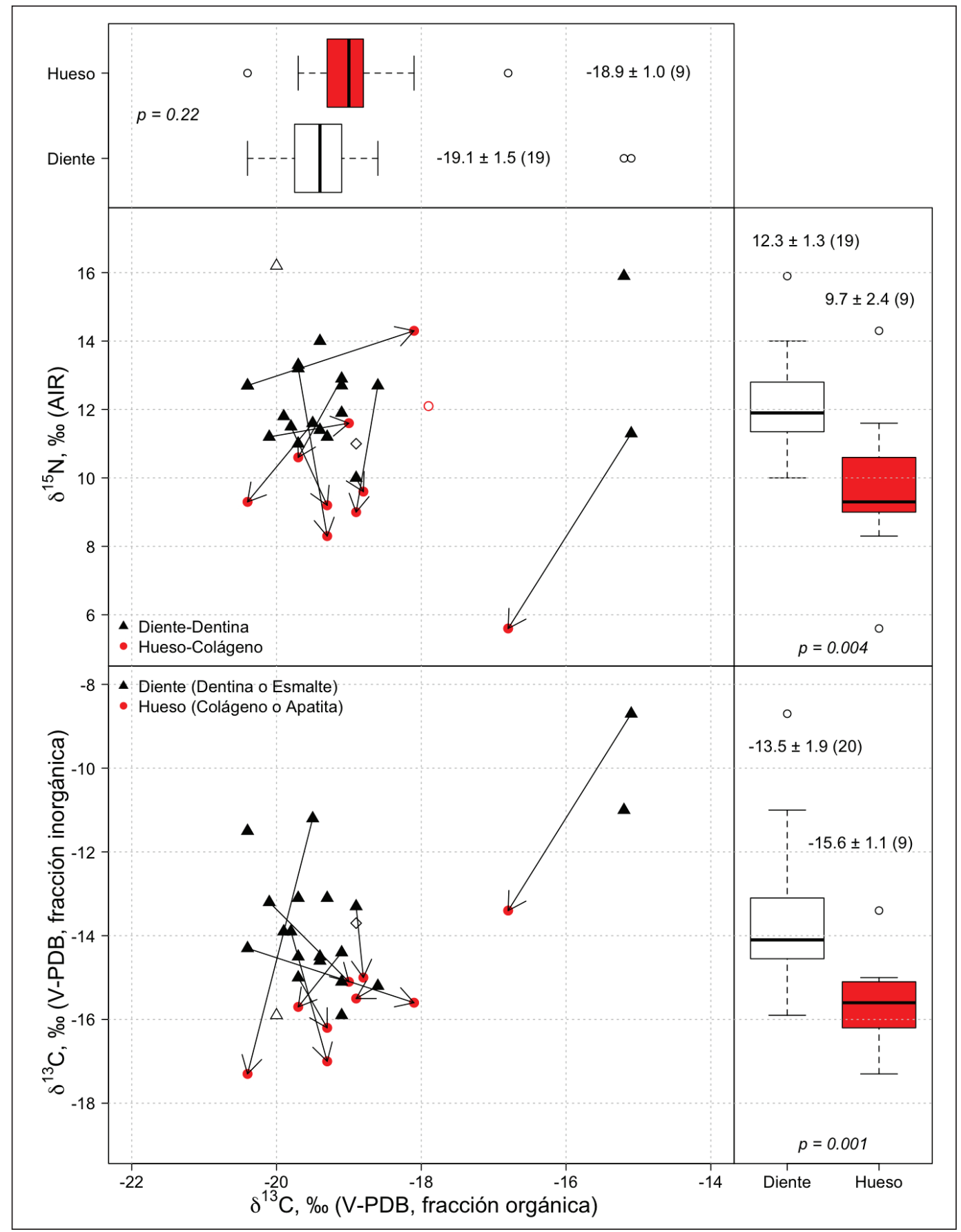

Fig. 3. Comparación de la composición isotópica de dientes y huesos para muestras del cementerio de la misión. La fracción inorgánica corresponde a esmalte (diente) y apatita (hueso), mientras que la fracción orgánica corresponde a dentina (diente) y colágeno (hueso). Las transiciones alimentarias de los nueve pares se representan con flechas. Los valores de los individuos con haplogrupo mitocondrial europeo se presentan en círculo rojo con centro blanco (colágeno) o en rombo (diente). Los valores de isótopos del diente deciduo [E 14-15 (1)] se presentan como triángulos vacíos en ambos gráficos. Los boxplots marginales comparan los valores de isótopos estables entre ambos tipos de muestra. Se prestan las medias $\pm 1 \sigma$ (tamaño muestral), y el valor de p luego del test de suma de rangos Wilcoxon para muestras no pareadas. 
$\left(\delta^{15} \mathrm{~N}_{\mathrm{d}} ; 12.3 \pm 1.3 \%\right.$, $\mathrm{n}=19$; Fig. 3). Mientras que los valores de $\delta^{13} \mathrm{C}_{\mathrm{c}} \mathrm{y}$ los de carbono en dentina $\left(\delta^{13} C_{d} ;-19.1 \pm 1.5 \%\right.$, $\left.n=19\right)$ no se distinguieron significativamente $(\mathrm{W}=111, \mathrm{p}=0.22$; Fig. 3 ). Sin embargo, sí detectamos diferencias significativas $(\mathrm{W}=21, \mathrm{p}=0.001)$ para la comparación de las fracciones inorgánicas entre los valores de carbono en apatita $\left(\delta^{13} \mathrm{C}_{\mathrm{a}}\right)$ y en esmalte $\left(\delta^{13} \mathrm{C}_{\mathrm{e}}\right.$ : $-13.5 \pm 1.9 \%$, n=19; Fig. 3).

La comparación directa de los valores isotópicos para los individuos a través de su vida resultó en los mismos patrones que al comparar huesos con dientes dentro de la misión como se expresó en el párrafo anterior. Estas transiciones alimentarias reflejadas en la diferencia entre los valores de huesos y dientes se representan con flechas en la Figura 3. Reconocemos que debido al bajo número de muestras pareadas con valores tanto en hueso como en diente las comparaciones quizá no tengan el poder estadístico suficiente ( $\mathrm{n}=9$ pares). Observamos que los valores de $\delta^{15} \mathrm{~N}_{\mathrm{c}}$ resultaron más bajos que de los de $\delta^{15} \mathrm{~N}_{\mathrm{d}}$ (test de suma de rangos Wilcoxon para muestras pareadas; $\mathrm{V}=4.5, \mathrm{p}=0.04, \delta^{15} \mathrm{~N}_{\mathrm{c}-\mathrm{d}}=-2.2 \pm 2.4 \%$ ), los valores de $\delta^{13} \mathrm{C}_{\mathrm{c}}$ no resultaron diferentes de los de $\delta^{13} \mathrm{C}_{\mathrm{d}}\left(\mathrm{V}=24, \mathrm{p}=0.91, \delta^{13} \mathrm{C}_{\mathrm{c}-\mathrm{d}}=0.1 \pm 1.2 \%\right.$ ) , mientras que los valores de $\delta^{13} \mathrm{C}_{\mathrm{a}}$ resultaron más bajos que los de $\delta^{13} \mathrm{C}_{\mathrm{e}}(\mathrm{V}=0, \mathrm{p}=0.01$, $\delta^{13} \mathrm{C}_{\mathrm{a}-\mathrm{e}}=-2.4 \pm 1.9 \%$ ).

Los valores de $\delta^{15} \mathrm{~N}_{\mathrm{d}}$ de la misión resultaron significativamente más bajos que los valores de $\delta^{15} \mathrm{~N}_{\mathrm{c}}$ para muestras pre-contacto de NIG $\left(\mathrm{W}=225, \mathrm{p}=0.03\right.$; Tabla 2). Los valores de $\delta^{13} \mathrm{C}_{\mathrm{d}}$ del cementerio también resultaron más bajos que aquellos de colágeno para muestras pre-contacto de NIG ( $\mathrm{W}=287, \mathrm{p}<0.002$; Tabla 2). En el caso de la fracción inorgánica de dientes $\left(\delta^{13} \mathrm{C}_{\mathrm{e}}\right)$ del cementerio y de huesos $\left(\delta^{13} \mathrm{C}_{\mathrm{a}}\right)$ de pre-contacto en la misma región, las diferencias fueron no significativas ( $\mathrm{W}=67, \mathrm{p}=0.84$; Tabla 2).

\section{DISCUSIÓN Y CONCLUSIONES}

En este trabajo propusimos analizar cambios en la dieta de los Selk'nam vinculados al proceso del contacto interétnico en el norte de Tierra del Fuego a través del uso de isótopos estables. Evaluamos las diferencias en los valores isotópicos, entre individuos exhumados en el cementerio de la Misión Salesiana "Nuestra Señora de La Candelaria" e individuos de períodos precontacto recuperados en PA. Nuestra hipótesis de trabajo fue que durante este periodo misional los cambios en la forma de vida de los individuos originarios en la misión se caracterizaron por una fuerte disminución del consumo proporcional de proteína animal local, acompañada por un gran incremento en el consumo de hidratos de carbono importados. Nuestros datos son consistentes con esta hipótesis y se ajustan a las predicciones; valores de $\delta^{15} \mathrm{~N}$ y de $\delta^{13} \mathrm{C}$ más bajos para individuos de la misión como respuesta al mayor consumo proporcional de harinas y otras plantas $\mathrm{C}_{3}$ en detrimento de una baja en el consumo de guanaco y otros animales locales.

El valor (estadístico) de las comparaciones realizadas depende de la calidad de los datos usados. Para los datos publicados en muchos casos debemos asumir que trabajamos con valores isotópicos sobre restos no alterados ya que en varios de los trabajos originales no se reportan parámetros para su evolución (por ejemplo $\% \mathrm{~N}$, $\% \mathrm{C}, \mathrm{C} / \mathrm{N}$ ). Esto no siempre es una falta de los autores, a veces es información que el laboratorio donde se realizan los análisis no provee o provee sólo parte de la misma, como fue tam-

TABLA 2. Comparación entre los valores isotópicos de hueso para muestras pre-contacto del Norte de la Isla Grande de Tierra del Fuego y de dientes de individuos exhumados en el cementerio de la misión Salesiana

\begin{tabular}{cccc}
\hline Isótopo & Hueso pre-NIG & Diente LMS & Test \\
\hline$\delta^{13} \mathrm{C}$ (orgánico) & $-17.4 \pm 2.1(18)$ & $-19.1 \pm 1.5(19)$ & $\mathrm{p}<0.002, \mathrm{~W}=287$ \\
$\delta^{15} \mathrm{~N}$ & $13.6 \pm 1.7(18)$ & $12.3 \pm 1.3(19)$ & $\mathrm{p}=0.03, \mathrm{~W}=225$ \\
$\delta^{13} \mathrm{C}$ (inorgánico) & $-14.0 \pm 1.6(6)$ & $-13.5 \pm 1.9(20)$ & $\mathrm{p}=0.84, \mathrm{~W}=0.84$ \\
\hline
\end{tabular}

NIG:Norte de la Isla Grande de Tierra del Fuego; LMS:misión Salesiana. Para cada población se presentan los valores medios $\pm 1 \sigma$ (tamaño de muestra). Los resultados estadísticos corresponden al test de suma de rangos de Wilcoxon para muestras no pareadas. 
bién nuestro caso en las muestras de hueso del cementerio. Sin embargo, el objetivo de este trabajo no es hacer una evaluación crítica de la calidad de los valores publicados si no usarlos como referencia para comparar aquellos de la Misión Salesiana.

Otro factor importante a tener en cuenta en estas comparaciones es que se trata de muestras de períodos pre y post-revolución industrial (cuando se inicia la liberación a la atmósfera de $\mathrm{CO}_{2}$ proveniente de combustibles fósiles) y por tanto los valores de $\delta^{13} \mathrm{C}$ atmosférico de ambos períodos son diferentes [efecto Suess, (Keeling, 1979)]. Debido a esto es que se debería aplicar una corrección a los valores medidos para hacerlos comparables. Sin embargo, desconocemos el año de muerte de cada individuo ya que los mismos fueron recuperados en un sector del cementerio en el cual no existen en la actualidad estructuras funerarias con dicha información (García Laborde, 2011), y sólo sabemos que el cementerio de la Misión Salesiana funcionó desde 1897 a 1948. Por tanto aplicando una corrección de -0.005 por año (Francey et al., 1999) nos da un factor de corrección mínimo de $-0.18 \%$ y un máximo de $-0.44 \%$ para este rango temporal. De cualquier manera, aun aplicando el factor máximo los valores de $\delta^{13} \mathrm{C}$ para la misión, estos no se verían desplazados lo suficiente como para alterar los resultados de este estudio, por ejemplo: el valor medio $\delta^{13} \mathrm{C}_{\mathrm{c}}$ para muestras del cementerio es $-18.9 \%$, aplicando la máxima corrección lo llevaríamos a $-18.5 \%$ que sigue siendo considerablemente más bajo que el promedio $\delta^{13} \mathrm{C}_{\mathrm{c}}$ para muestras pre-contacto de NIG (-17.4\%o). Además sabemos que el período con mayor densidad de defunciones fue de 1900 a 1903 (Casali, 2011), por tanto aplicar estos factores extremos a todas las muestras analizadas, probablemente tampoco nos brindaría una mejor aproximación a sus valores reales.

Como ya ha sido publicado en varias ocasiones, la dieta de los cazadores-recolectores de PA para momentos pre-contacto estaba comprendida por la dicotomía entre los ambientes y recursos marinos y terrestres (Borrero et al., 2001; Borrero y Barberena, 2006; Borrero et al., 2009; Guichón et al., 2001, 2006; Panarello et al., 2006; Santiago et al., 2011; Schinder y Guichón, 2003; Schinder et al., 2003; Suby y Guichón, 2009; Tafuri et al., 2017; Tessone et al., 2003; Yesner et al., 1991, 2003, Zangrando et al., 2014, 2004). Esto se hace evidente en las fuertes correlaciones entre los valores de nitrógeno y carbono de colágeno, y los de carbono para colágeno y apatita generadas por estos dos puntos extremos de la distribución isotópica de los recursos [isotopic end-members, (Fry, 2006)]. En nuestro metaanálisis de las distintas regiones de PA detectamos diferencias estadísticas notables entre las regiones definidas. Vemos que el Norte de la Isla Grande de Tierra del Fuego, nuestra región de interés, se caracteriza por una dieta basada en proteínas animales de origen terrestre $\mathrm{C}_{3}$ (principalmente guanaco) con cierto grado de dietas mixtas, que según Schinder y colaboradores podrían llegar a incluir hasta un $50 \%$ de recursos marinos en algunos casos (Schinder et al., 2003).

Nuestros análisis muestran que, en promedio, los individuos de la misión tienen valores isotópicos más bajos tanto en colágeno como en apatita en comparación con aquellas muestras obtenidas en la misma región geográfica (NIG) pero correspondientes a períodos pre-contacto. Esta diferencia o disminución en los valores de $\delta^{15} \mathrm{~N}$ respondería directamente a un menor consumo proporcional de proteína animal, o quizá a la introducción de proteína animal de diferente origen, pero con valores más bajos. Para esta población de cazadores-recolectores, esto representaría directamente el remplazo del guanaco como fuente proteínica principal, y quizá el abandono de ítems de la dieta que contribuirían a una dieta mixta.

Si bien los cazadores-recolectores del NIG ya consumían primariamente una dieta $\mathrm{C}_{3}$ la disminución de los valores de $\delta^{13} \mathrm{C}$ y su desplazamiento hacia valores más bajos dentro del rango $\mathrm{C}_{3}$ podría explicarse por factores relacionados al origen del carbono consumido: 1- la disminución en el consumo de guanaco podría también reducir los valores de $\delta^{13} \mathrm{C}$, ya que los animales tienden a tener valores de $\delta^{13} \mathrm{C}$ mayores que las plantas que estos consumen aunque la diferencia suele ser pequeña (Fry, 2006); 2- el abandono o reducción del consumo de los complementos marinos traería aparejado definitivamente una reducción en los valores de $\delta^{13} \mathrm{C} ; 3$ - el gran incremento proporcional del consumo de nuevas especies $\mathrm{C}_{3}$ como el trigo, el arroz y la papa, importadas de regiones con climas con- 
ducentes a una mayor discriminación isotópica (por tanto, con valores más bajos (Cerling et al., 1997; Farquhar, Ehleringer y Hubick, 1989) desplazarían los valores de $\delta^{13} \mathrm{C}$ de los consumidores. El hecho de que la disminución de los valores se dé en colágeno y en apatita, sugiere que tanto la ingesta de proteína como de lípidos/ grasas e hidratos de carbono, es decir la dieta como un todo, sufrió este cambio.

Una de las problemáticas que enfrentamos para poder interpretar la transición alimentaria es la cronología de las muestras, es decir, qué momentos de la misión están reflejados en estos datos (por ejemplo, si las muestras corresponden a los inicios o al final del uso del cementerio). La misión introdujo cambios profundos y rápidos en la vida de estos individuos, pero inclusive estos cambios no fueron constantes, al menos en lo que se refiere a dieta. Como lo describe (Casali, 2011), los primeros años de la misión (18931896) fueron de transición, con asentamientos esporádicos de indígenas y consumo ocasional de alimento provisto por los misioneros, luego hasta 1903 hubo un gran incremento en el consumo de hidratos de carbono (particularmente harina, arroz, papas y azúcar), y hubo un aumento en el consumo de ovejas, aunque el consumo de harina se mantuvo elevado.

Además, desconocemos si las personas inhumadas en el cementerio vivieron varios años o sólo unas semanas en la misión, y por tanto la señal isotópica de sus tejidos (hueso principalmente) puede que no haya alcanzado un equilibrio isotópico con la dieta de la misión. La adquisición de la señal isotópica de esta nueva (aunque variable) dieta no es inmediata en los tejidos óseos. La tasa de recambio del colágeno es baja y puede llevar años adquirir completamente una nueva señal isotópica. En principio, podríamos estar viendo muestras con valores intermedios o inclusive quizá ninguno con el valor de "equilibrio final" con la nueva dieta. De cualquier manera, que se vean diferencias entre los grupos de muestras (pre $v s$. post) sugiere que el cambio fue muy grande en cuanto a la señal isotópica, la proporción de la dieta y/o el tiempo consumiendo la misma antes de la muerte. Esta variabilidad se ve reflejada en algunos individuos de la misión muy diferentes de los precontacto y algunos con valores similares.

Resultan interesantes las comparaciones den- tro de la misión donde podemos ver dos etapas en la vida de las personas que allí vivieron. Tanto la comparación poblacional hueso vs. diente como aquella sobre muestras del mismo individuo (muestras pareadas) sugieren que, en promedio, la dieta de los individuos durante su niñez o juventud habría sido diferente tanto con respecto a los momentos pre-contacto como a la dieta de los adultos en la misión. Aunque con esta muestra tan pequeña es muy difícil hacer mayores inferencias, se detectan posibles transiciones alimentarias en la vida de algunos individuos. En principio, los datos sugieren que el consumo proporcional de proteína animal en la niñez sería intermedio entre los individuos adultos pre y post-contacto. Poder hacer mayores interpretaciones sobre qué representa esta dieta de los individuos en su niñez o juventud es complejo pues hay un buen número de factores desconocidos. Por tanto, la interpretación de una dieta diferencial e intermedia debe tomarse con cautela y teniendo en cuenta ciertos aspectos de la muestra con la que trabajamos, principalmente su heterogeneidad. En primer lugar y sumado a lo expresado anteriormente sobre la temporalidad de las muestras, también desconocemos si estos individuos nacieron y pasaron sus primeros años dentro o fuera de la misión; podríamos asumir lo primero para los más jóvenes, pero quizá no para los de mayor edad. En segundo lugar, no todos los dientes proveen la misma información temporal, algunos de los dientes muestreados son de formación temprana (canino, incisivo) y por lo tanto quizá sus valores estén parcialmente influenciados por la dieta materna y la lactancia (Beaumont y Montgomery, 2015). Lo mismo podría decirse de los dientes de formación intermedia (premolar, segundo molar) aunque en un menor grado (Beaumont y Montgomery, 2015). Sin embargo, no detectamos diferencias de acuerdo con la edad del individuo, y el tipo de diente no parece influir en sus valores isotópicos, a excepción del diente deciduo de E 14-15(1). En tercer lugar, y relacionado al punto anterior, la influencia del metabolismo nutricional durante el crecimiento de una persona sobre los valores isotópicos en sus tejidos no ha sido cuantificada apropiadamente como para poder atribuirle las diferencias observadas (Fuller et al., 2005).

A pesar del efecto desconocido de los factores antes mencionados, vemos sin lugar a dudas 
que los individuos exhumados en el cementerio de la misión tenían una dieta diferente a aquellos pre-contacto de la Isla de Tierra del Fuego. Esto se contrapone con los resultados de Tafuri et al. (2017), quienes observan una uniformidad en los valores isotópicos para poblaciones prey post-contacto del Canal Beagle, atribuyéndola a la resiliencia y resistencia de estos grupos de cazadores-recolectores ante la influencia de los europeos. Si el cambio hacia nuevas fuentes de alimentación fue similar (en el tipo de alimentos nuevos, su cantidad y su tiempo de consumo) tanto en el norte de la isla como en el CB, las diferencias isotópicas entre pre y post-contacto deberían ser mayores para los individuos del CB debido a su dieta tradicional, preponderantemente marina. Sin embargo, esto no se ve reflejado en los datos. Entonces, la diferencia entre la respuesta de las poblaciones del norte y del sur de Tierra del Fuego frente a las potenciales transiciones alimentarias podría deberse a numerosos factores, pero no contamos con información suficiente para poder evaluarlos en este trabajo. Queremos, sin embargo, destacar uno que nos parece fundamental: los restos humanos presentados por Tafuri y colaboradores (Tafuri et al., 2017) correspondientes al período post-contacto tienen como fechas más recientes algunas cercanas al 1800, mientras que en el cementerio Salesiano las personas fueron inhumadas entre 1897 y 1948. Si bien la diferencia temporal podría ser menor a un siglo, se trata de un período de rápida expansión de las estancias y del estado-nación en la Isla y corresponde a un período de cambios drásticos en las poblaciones nativas. Una diferencia importante es que los restos de los individuos exhumados en la misión Salesiana correspondían a miembros de la comunidad Selk'nam que quizás habían sido integrados completamente a la vida diaria de la misión, mientras que los restos post-contacto del $\mathrm{CB}$ correspondían a miembros de la comunidad Yámana pero que no habían asimilado completamente las costumbres y dieta de los misioneros anglicanos (y resistieron como lo propone Tafuri et al. (2017). Lamentablemente una gran proporción de los restos analizados por Tafuri y sus colaboradores provienen de colecciones poco contextualizadas y muy poco ordenadas (Marangoni et al., 2011), por tanto es difícil trazar un paralelo de estos individuos con los de la misión Salesiana para así comparar los efectos de la colonización en los dos extremos de la Isla de Tierra del Fuego.

Este trabajo remarca que los estudios de los cementerios asociados a las misiones resultan de suma importancia dado que las muestras de allí recuperadas proveen información única acerca de los cambios en la alimentación y/o modos de subsistencia de las poblaciones nativas en uno de los momentos particulares del contacto interétnico (Bybee, 2004; García Laborde, 2011; García Laborde et al., 2010; Larsen et al., 2001). Además destaca que estudiar procesos complejos, como lo fue el contacto interétnico, contando con el registro bioarqueológico al mismo tiempo que los datos históricos y las fuentes escritas permite la contrastación de hipótesis que de otra manera sería muy difícil lograr. Particularmente el análisis de los isótopos estables de carbono y nitrógeno permitió validar el cambio en la alimentación y las formas de vida descrito por las fuentes escritas y sugerido por otras evidencias del registro arqueológico (por ejemplo empeoramiento de la salud a través de estudios osteológicos y/o paleopatológicos). De esta complejidad, quedan sin dudas aristas por entender, por ejemplo la variabilidad isotópica interna a la misión, la cual podría evaluarse a futuro mediante estudios secuenciales de isótopos estables sobre dentina que permita definir la historia nutricional de estos individuos.

\section{AGRADECIMIENTOS}

Los autores quieren agradecer a Josefina Motti, Marilina Martucci, Patricia Palacio, Rocío Guichón, Mariana Segura y Alejandra Ibáñez, por su colaboración y apoyo en el trabajo tanto en el campo como en el laboratorio. A las autoridades de la escuela Agrotécnica La Candelaria y de la orden Salesiana. A las autoridades provinciales y municipales; y especialmente a los miembros de la comunidad "Selk' nam" residentes en Rio Grande por su interés y apoyo en el proyecto. A los dos evaluadores/as anónimos/ as por sus valiosos comentarios.

\section{LITERATURA CITADA}

Ambrose, S. H. (1990). Preparation and characterization of bone and tooth collagen for isotopic analysis. Journal of Archaeological Science, 17(4), 431-451. doi:10.1016/0305-4403(90)90007-R 
Ambrose, S. H. y DeNiro, M. J. (1986). Reconstruction of African human diet using bone collagen carbon and nitrogen isotope ratios. Nature, 319(6051), 321-324. doi: $10.1038 / 319321 \mathrm{a} 0$

Barberena, R. (2004). Arqueología e isótopos estables en Tierra del Fuego. En L. A. Borrero y R. Barberena (Compiladores), Temas de Arqueología. Arqueología del norte de la Isla Grande de Tierra del Fuego (pp. 135-169). Buenos Aires, Argentina: Dunken.

Bass, W. M. (1995). Human osteology: a laboratory and field manual (Missouri Archaeological Society, Special Publications, No. 2). Columbia, Missouri, U.S.A.: Missouri Archaeological Society. Recuperado de https:// www.biblio.com/book/human-osteology-laboratoryfield-manual-missouri/d/895424540

Beaumont, J. y Montgomery, J. (2015). Oral histories: a simple method of assigning chronological age to isotopic values from human dentine collagen. Annals of $\mathrm{Hu}$ man Biology, 42(4), 407-414.

Bello, S. M., Thomann, A., Signoli, M., Dutour, O. y Andrews, P. (2006). Age and sex bias in the reconstruction of past population structures. American Journal of Physical Anthropology, 129(1), 24-38. doi:10.1002/ ajpa. 20243

Borrero, L. A. (1992). El registro arqueológico del contacto: enfermedad y discontinuidad poblacional. Trabajo presentado en Simposio Encuentro de dos Culturas. Centro Cultural General San Martín, Buenos Aires, Argentina (pp. 1-6).

Borrero, L. A. (2001a). El poblamiento de la Patagonia: toldos, milodones y volcanes. Buenos Aires, Argentina: Emece Editores.

Borrero, L. A. (2001b). Los selk'nam: (Onas): evolución cultural en Tierra del Fuego. Buenos Aires, Argentina: Galerna.

Borrero, L. A. y Barberena, R. (2006). Hunter-gatherer home ranges and marine resources: an archaeological case from southern Patagonia. Current Anthropology, 47(5), 855-867.

Borrero, L. A., Barberena, R., Franco, N. V., Charlin, J. y Tykot, R. H. (2009). Isotopes and rocks: geographical organization of southern Patagonian hunter-gatherers. International Journal of Osteoarchaeology, 19(2), 309327. doi:10.1002/oa.1036

Borrero, L. A., Guichon, R. A., Tykot, R., Kelly, J., Prieto Iglesias, A. y Cárdenas, P. (2001). Dieta a partir de isótopos estables en restos óseos humanos de Patagonia Austral. Estado actual y perspectivas. Anales del Instituto de la Patagonia. serie Cs Hs, 29, 119-127. Recuperado de http://bibliotecadigital.umag.cl/handle/20.500.11893/1521

Boutton, T. W., Lynott, M. J. y Bumsted, M. P. (1991). Stable carbon isotopes and the study of prehistoric human diet. Critical Reviews in Food Science and Nutrition, 30(4), 373-385.

Brooks, S. y Suchey, J. (1990). Skeletal age determination based on the os pubis: a comparison of the AcsádiNemeskéri and Suchey-Brooks methods. Human Evolution, 5(3), 227-238. doi:10.1007/bf02437238

Buikstra, J. E. y Mielke, J. L. (1985). Demography, diet, and health. En R. I. Gilbert y J. H. Mielke (Ed.), Analysis of prehistoric diets (pp. 360-422). London, United Kingdom: Academic Press.

Buikstra, J. E. y Ubelaker, D. H. (1994). Standards for data collection from human skeletal remains. Proceedings of a seminar at the Field Museum of Natural History. Recuperado de http://core.tdar.org/document/50180/ standards-for-data-collection-from-human-skeletal- emains-proceedings-of-a-seminar-at-the-field-museumof-natural-history

Bybee, A. (2004). Bioanthropological investigations of historic cemeteries: what can we learn from biological, cultural,and mortuary remains? $5^{\text {th }}$ Annual Council for West Virginia Archeology Spring Workshop, West Virginia, USA.

Casali, R. (2011). Contacto interétnico en el norte de Tierra del Fuego: La Misión Salesiana La Candelaria (Rio Grande) y la salud de la población Selk'nam (18951931) (Tesis doctoral). Universidad Nacional de Mar del Plata, Mar del Plata.

Casali, R., Fugassa, M. H. y Guichón, R. A. (2006). Aproximación epidemiológica al proceso de contacto interétnico en el norte de Tierra del Fuego. Magallania (Punta Arenas), 34(001), 87-101.

Cerling, T. E., Harris, J. M., MacFadden, B. J., Leakey, M. G., Quade, J., Eisenmann, V. y Ehleringer, J. R. (1997). Global vegetation change through the Miocene/Pliocene boundary. Nature, 389(6647), 153-158. doi: $10.1038 / 38229$

Clementz, M. T., Fox-Dobbs, K., Wheatley, P. V., Koch, P. L. y Doak, D. F. (2009). Revisiting old bones: coupled carbon isotope analysis of bioapatite and collagen as an ecological and palaeoecological tool. Geological Journal, 44(5), 605-620. doi:10.1002/gj.1173

Cunningham, C., Scheuer, L. y Black, S. (2000). Developmental Juvenile Osteology. San Diego, USA: Academic Press.

DeNiro, M. J. (1985). Postmortem preservation and alteration of in vivo bone collagen isotope ratios in relation to palaeodietary reconstruction. Nature, 317(6040), 806809. doi:10.1038/317806a0

DeNiro, M. J. y Epstein, S. (1978). Influence of diet on the distribution of carbon isotopes in animals. Geochimica et Cosmochimica Acta, 42(5), 495-506. doi:10.1016/00167037(78)90199-0

DeNiro, M. J. y Epstein, S. (1981). Influence of diet on the distribution of nitrogen isotopes in animals. Geochimica et Cosmochimica Acta, 45(3), 341-351. doi:10.1016/0016-7037(81)90244-1

DeNiro, M. J. y Schoeninger, M. J. (1983). Stable carbon and nitrogen isotope ratios of bone collagen: variations within individuals, between sexes, and within populations raised on monotonous diets. Journal of Archaeological Science, 10(3), 199-203. doi:10.1016/03054403(83)90002-X

Farquhar, G. D., Ehleringer, J. R. y Hubick, K. T. (1989). Carbon isotope discrimination and photosynthesis. Annual Review of Plant Physiology and Plant Molecular Biology, 40(1), 503-537. doi:10.1146/annurev. pp.40.060189.002443

Fazekas, I. G. y Kosa, F. (1978). Forensic fetal osteology. Budapest, Hungría: Akadémiai Kiadó.

Francey, R. J., Allison, C. E., Etheridge, D. M., Trudinger, C. M., Enting, I. G., Leuenberger, M., Langenfelds, R. L., Michel, E. y Steele, L. P. (1999). A 1000-year high precision record of $\delta 13 \mathrm{C}$ in atmospheric $\mathrm{CO} 2$. Tellus $B$, 51(2), 170-193. doi:10.1034/j.1600-0889.1999.t01-100005.x

Fry, B. (2006). Stable isotope ecology. New York, USA: Springer. Recuperado de http://www.springerlink.com/ content/978-0-387-30513-4/contents/

Fuller, B. T., Fuller, J. L., Sage, N. E., Harris, D. A., O'Connell, T. C. y Hedges, R. E. M. (2005). Nitrogen balance and delta $15 \mathrm{~N}$ : why you're not what you eat during nutritional stress. Rapid Communications in Mass Spectrometry, 19(18), 2497-2506. doi:10.1002/rcm.2090 
García Laborde, P. (2011). El cementerio de la antigua misión Salesiana "Nuestra Señora de La Candelaria" (Rio Grande, Tierra del Fuego) Bioarqueología de contacto en el siglo $X I X-X X$ (Tesis de pregrado). Universidad Nacional del Centro de la Provincia de Buenos Aires. Olavarría.

García Laborde, P. (2017). Estado nutricional de la población Selk'nam: aproximación bioarqueológica al impacto generado por la misionalización. Misión Salesiana Nuestra Señora de La Candelaria, Tierra del Fuego (Siglos XIX-XX (Tesis doctoral). Universidad Nacional del Centro de la Provincia de Buenos Aires. Olavarría.

García Laborde, P., Guichón, R. A. y González, N. G. (2015). Una aproximación tafonómica al antiguo cementerio de la Misión Salesiana de Río Grande, Tierra del Fuego. Arqueología, 21(2), 277-290.

García Laborde, P., Suby, J. A., Guichón, R. A. y Casali, R. (2010). El antiguo cementerio de la misión de Río Grande, Tierra del Fuego. Primeros resultados sobre patologías nutricionales-metabólicas e infecciosas/The ancient cemetery of Río Grande Mision, Tierra del Fuego: nutritional-metabolic and infectious diseases. Revista Argentina de Antropología Biológica, 12(1), 57-69.

González, M. E. (2007). Estudios de interés tafonómico en los restos óseos humanos de Laguna Tres Reyes 1 (Partido de Adolfo Gonzales Chaves, provincia de Buenos Aires). Intersecciones en Antropología, 8, 215-233.

Guichón, R. A., Borrero, L. A., Prieto, A. I., Cárdenas, P. y Tykot, R. H. (2001). Nuevas determinaciones de isótopos estables para Tierra del Fuego. Revista Argentina de Antropología Biológica, 3(1), 113-126.

Guichón, R. A., Suby, J. A., Casali, R. y Fugassa, M. H. (2006). Health at the time of Native-European contact in Southern Patagonia: first steps, results, and prospects. Memórias do Instituto Oswaldo Cruz, 101 (2), 97-105.

Gusinde, M. (1990). Los indios de Tierra del Fuego. Los Selk'nam. Buenos Aires: Centro Argentino de Etnología Americana.

Hedges, R. E. M. y Reynard, L. M. (2007). Nitrogen isotopes and the trophic level of humans in archaeology. Journal of Archaeological Science, 34(8), 1240-1251. doi:10.1016/j.jas.2006.10.015

Keeling, C. D. (1979). The Suess effect: ${ }^{13}$ Carbon- ${ }^{14}$ Carbon interrelations. Environment International, 2(4-6), 229300. doi:10.1016/0160-4120(79)90005-9

Kellner, C. M. y Schoeninger, M. J. (2007). A simple carbon isotope model for reconstructing prehistoric human diet. American Journal of Physical Anthropology, 133(4), 1112-1127. doi:10.1002/ajpa.20618

Kelly, J. F. (2000). Stable isotopes of carbon and nitrogen in the study of avian and mammalian trophic ecology. Canadian Journal of Zoology, 78(1), 1-27. doi:10.1139/cjz-78-1-1

Krogman, W. M. y İșcan, M. Y. (1986). The human skeleton in forensic medicine. Springfield, USA: C.C. Thomas.

Larsen, C., Griffin, M. C., Hutchinson, D. L., Noble, V. E., Norr, L., Pastor, R. F., Ruff, C. B., Russell, K. F., Schoeninger, M. J., Schultz, M., Simpson, S. W. y Teaford M.F. (2001). Frontiers of contact: bioarchaeology of Spanish Florida. Journal of World Prehistory, 15(1), 69-123. doi:DOI: 10.1023/A:1011180303211

Luz, B. y Kolodny, Y. (1985). Oxygen isotope variations in phosphate of biogenic apatites. IV. Mammal teeth and bones. Earth and Planetary Science Letters, 75(1), 2936. doi:10.1016/0012-821X(85)90047-0

Mann, R. W., Jantz, R. L., Bass, W. M. y Willey, P. S. (1991). Maxillary suture obliteration: a visual method for estimating skeletal age. Journal of Forensic Sciences, 36(3), 781-791.
Marangoni, A., Belli, M. L., Caramelli, D., Moggi Cecchi, J., Zavattaro, M. y Manzi, G. (2011). Tierra del Fuego, its ancient inhabitants, and the collections of skeletal remains in the Museum of Anthropology of Florence and Rome. Museologia Scientifica, 5(1-2).

Martucci, M. (2016). Heterogeneidad espacial en la misión salesiana Nuestra Señora de La Candelaria: expresión de la identidad étnica Selk'nam durante el proceso de contacto interétnico (Río Grande, Tierra del Fuego) (Tesis doctoral). Universidad Nacional del Centro de la Provincia de Buenos Aires. Olavarria.

Meindl, R. y Loveyoy, C. (1989). Age Changes in the Pelvis: Implications for Paleodemography. En M. Iscan (Ed.), Age Markers in the Human Skeleton (pp. 137-168). Springfield, USA: Charles C. Thomas.

Motti, J., Harkins, P., García Laborde, P., Valenzuela, L. O., Cuello, M., Stone, A., Bravi, C. M. y Guichón, R. A. (2015). Develando el poblamiento humano de la $\mathrm{Pa}$ tagonia austral: aportes de los estudios de secuencias completas de ADN mitoncondrial. En M Kowalewski y M Raño (Ed.) .Libro de Resúmenes de las XII Jornadas Nacionales de Antropología Biológica p. 32). Corrientes: Asociación Argentina de Antropología Biológica.

Panarello, H., Zangrando, F. A., Tessone, A., Kozameh, L. y Testa, N. (2006). Análisis comparativo de paleodietas humanas entre la región del canal Beagle y Península Mitre: Perspectivas desde los isótopos estables. $\mathrm{Ma}$ gallania (Punta Arenas), 34(2), 37-46. doi:10.4067/ S0718-22442006000200004

Peterson, B. J. y Fry, B. (1987). Stable isotopes in ecosystem studies. Annual Review of Ecology and Systematics, 18(1), 293-320. doi:10.1146/annurev. es.18.110187.001453

Petzke, K. J., Boeing, H., Klaus, S. y Metges, C. C. (2005). Carbon and nitrogen stable isotopic composition of hair protein and amino acids can be used as biomarkers for animal-derived dietary protein intake in humans. Journal of Nutrition, 135(6), 1515-1520.

Petzke, K. J., Fuller, B. T. y Metges, C. C. (2010). Advances in natural stable isotope ratio analysis of human hair to determine nutritional and metabolic status. Current Opinion in Clinical Nutrition and Metabolic Care, 13(5), 532-540. doi:10.1097/MCO.0b013e32833c3c84

R Development Core Team. (2008). R: A language and environment for statistical computing. Vienna, Austria: $\mathrm{R}$ Foundation for Statistical Computing. Recuperado de from http://www.R-project.org

Ramenofsky, A. F. (1988). Vectors of death: the archaeology of European contact. Albuquerque, USA: University of New Mexico Press.

Santiago, F., Salemme, M., Suby, J. y Guichón, R. A. (2011). Restos humanos en el norte de Tierra del Fuego: aspectos contextuales, dietarios y paleopatológicos. Intersecciones en Antropología, 12(1), 147-162.

Scheuer, L., Black, S. y Schaefer, M. C. (2008). Juvenile osteology: a laboratory and field manual. New York, USA: Academic Press.

Schinder, G. y Guichón, R. A. (2003). Isótopos estables y estilo de vida en muestras óseas humanas de Tierra del Fuego. Magallania (Punta Arenas), 31, 33-44.

Schinder, G., Guichón, R. A., Comparatore, V. y Burry, S. (2003). Inferencias paleodietarias mediante isótopos estables en restos óseos humanos provenientes de Tierra del Fuego, Argentina. Revista Argentina de Antropología Biológica, 5(2), 15-31.

Schutkowski, H. (1993). Sex determination of infant and juvenile skeletons: I. Morphognostic features. Ameri- 
can Journal of Physical Anthropology, 90(2), 199-205. doi:10.1002/ajpa.1330900206

Schwarcz, H. P. y Schoeninger, M. J. (1991). Stable isotope analyses in human nutritional ecology. American Journal of Physical Anthropology, 34(13), 283-321. doi:10.1002/ajpa.1330340613

Sponheimer, M., Robinson, T. F., Roeder, B.L, Passey, B. H., Ayliffe, L. K., Cerling, T. E., Dearing M. D. y Ehleringer, J. R. (2003). An experimental study of nitrogen flux in llamas: is $\mathrm{N}-14$ preferentially excreted? Journal of Archaeological Science, 30(12), 1649-1655.

Suby, J. A. y Guichón, R. A. (2009). Diet, nutrition and femoral robusticity of hunter-gatherers in southern Patagonia: experiences and perspectives. International Journal of Osteoarchaeology, 19(2), 328-336. doi:10.1002/oa.1040

Szilvassy, J. (1988). Altersdiagnose am skelett. En R. Knussmann (Ed.) (pp. 421-443). Stuttgart, Alemania: Fischer, G, Verlag.

Tafuri, M. A., Zangrando, A. F. J., Tessone, A., Kochi, S., Moggi Cecchi, J., Di Vincenzo, F., Profico, A. y Manzi, G. (2017). Dietary resilience among hunter-gatherers of Tierra del Fuego: isotopic evidence in a diachronic perspective. PloS One, 12(4), e0175594. doi:10.1371/ journal.pone. 0175594

Tessone, A., Zangrando, F. A., Valencio, S. y Panarello, H. (2003). Isótopos estables del carbono en restos óseos humanos de la región del canal Beagle, Isla Grande de Tierra del Fuego. Revista Argentina de Antropología Biológica, 5(2), 33-43.

Ubelaker, D. H. (1999). Human Skeletal Remains: Excava- tion, Analysis, Interpretation. Washington, USA: Taraxacum.

Yesner, D. R., Figuerero Torres, M., Guichón, R. A. y Borrero, L. A. (1991). Análisis de isótopos estables en esqueletos humanos: confirmación de patrones de subsistencia etnográficos para Tierra del Fuego. Shincal, 3(2), 182-191.

Yesner, D. R., Torres, M. J. F., Guichon, R. A. y Borrero, L. A. (2003). Stable isotope analysis of human bone and ethnohistoric subsistence patterns in Tierra del Fuego. Journal of Anthropological Archaeology, 22(3), 279291. doi:10.1016/S0278-4165(03)00040-0

Yoder, C. y Bartelink, E. (2010). Effects of differente sample preparation methods on stable carbon and oxygen isotope values of bine apattite: a comparison of two treatment protocols. Archaeometry, 52(1), 115-130.

Zangrando, A. F. J., Panarello, H. O., y Piana, E. L. (2014). Zooarchaeological and stable isotopic assessments on Pinniped-Human relations in the Beagle Channel (Tierra del Fuego, Southern South America). International Journal of Osteoarchaeology, 24(2), 231-244. doi:10.1002/oa.2352

Zangrando, A. F., Tessone, A., Valencio, S., Panarello, H., Mansur, M. y Salemme, M. (2004). Isótopos estables y dietas humanas en ambientes costeros. En M. J. Feliu Ortega, J. M. Calleja, M. C. Edreira Sánchez, M. C. Fernández Lorenzo, M. P. Martínez Brell, A. Gil Moreno y R. Alcántara Puerto (Ed.), Avances en Arqueometría 2003 (pp. 91-97). Universidad de Cádiz Servicio de Publicaciones. Recuperado de http://dialnet.unirioja.es/ servlet/articulo? codigo $=1091412$ 